\title{
The rumen and beyond: Nutritional physiology of the modern dairy cow ${ }^{1}$
}

\author{
D. M. Bravo and E. H. Wall ${ }^{2}$ \\ Pancosma, Geneva, $\mathrm{CH}-1218$ Switzerland
}

\section{INTRODUCTION}

Can knowledge originating from developmental physiology, nutritional immunology, or nutritional endocrinology in other species (humans, rodents, or other farm animals) have implications for ruminant nutrition? Would this new knowledge help break through the current paradigms of nutrition and production in ruminants and create a new one? Could unremitting issues (e.g., ketosis, acidosis) be reinterpreted within this new paradigm to help derive efficient new concepts? These questions were the fundamental subjects debated during the symposium "The rumen and beyond: Nutritional physiology of the modern dairy cow," held during the 2015 American Dairy Science Association-American Society of Animal Science Joint Annual Meeting in Orlando, Florida.

Ruminants live in symbiosis with a complex ecosystem hosted in their first digestive compartment, which enables them to benefit from nondigestible dietary compounds. The most obvious example is the rumen fermentation of nondigestible cellulose that produces volatile FA, an end product for the rumen bacteria and the main energy source of the host. Nutrition supported by nutritional research allowed a better understanding of the fermentation mechanisms so as to maximize its efficiency and the delivery of microbial nutrients to the host. These progresses accompanied the improvement of genotypes and enhanced production efficiency. The first speaker, VandeHaar et al. (2016), highlighted that refined nutritional programs, combined with genetic selection for milk production, and farm management have more than doubled feed efficiency during the last 100 yr. Nutritionally speaking, the increased efficiency was triggered by the dilution of maintenance requirements, allowing more energy to become available for milk production. This approach is reaching its limits, emphasizing the importance of novel strategies to con-

\footnotetext{
Received July 31, 2015.

Accepted August 4, 2015.

${ }^{1}$ Presented as part of the ADSA Production Division Symposium: The Rumen and Beyond - Nutritional Physiology of the Modern Dairy Cow at the ADSA-ASAS Joint Annual Meeting, Orlando, Florida, July 2015.

${ }^{2}$ Corresponding author: emma.wall@pancosma.ch
}

tinue to improve productivity. Individuals with a low residual feed intake eat similar amounts but produce more milk than others; their digestion, metabolism, or other nutrient-capturing functions are more efficient than their counterparts of similar size. VandeHaar et al. (2016) suggested that an understanding of the factors underpinning variations in residual feed intake could lead to novel strategies for improving milk production efficiency.

Considering its importance in cow production, it is not surprising that the rumen and its ecosystem have monopolized most nutritional research. This is reflected very well in nutritional requirements and concepts (e.g., the Cornell Net Carbohydrate and Protein System; http://cncps.cornell.edu/) and additive technologies for ruminants (e.g., the ionophore monensin, which shifts the rumen fermentation toward less acetate and more propionate that is energetically more profitable for the cow). This is a good example of how a paradigm (here, importance of the rumen microbiome synergy for delivering microbe-derived metabolites for the host) leads to the development of nutritional programs and to corresponding technologies. However, dairy scientists should shift their focus to include other organs and potential targets and conceptualize a new paradigm. For example, human nutrition and medicine are making great strides in establishing the gut as a cardinal organ of physiology. The gut is an intelligent sensory organ (Furness et al., 2013), permanently detecting its complex and ever-changing environment, processing the information and reacting to it. Alas, in ruminants, very few scientists have dedicated interest in the (lower) gut, for most view it as a simple tube with the minor task of further digesting and absorbing dietary or rumen-created compounds. A quick PubMed search (http://www. ncbi.nlm.nih.gov/pubmed) with the key words "gut," "ruminants," and "intestine" produced 123 papers in 2014, most of them not nutritionally relevant, whereas 521 articles focused on the rumen. In this context, the second speaker, Steele et al. (2016), suggested 3 areas for exploration: intestinal barrier function, effect of weaning on intestinal health and function, and gastrointestinal adaptations to dietary and environmental changes. In young ruminants, compromised gut epithelial barrier function increases susceptibility to diseases, 
mortality, and decreases lifetime productivity. Growth of gut tissue is critical and should be explored at the organ, cellular, and molecular levels to provide opportunities for improved animal performance via optimized gut barrier function. In adult ruminants, the authors suggested that acidosis and associated pathologies could be due to increased permeability of the rumen wall and inflammation in the lower gut. Developing this argument could lead to novel way of attenuation of acidosis consequences via new dietary concepts and interventions aimed at protecting total-tract barrier function.

The third speaker, Baumgard et al. (2015), emphasized this gut-oriented research axis linking disrupted gut function to physiological and environmental changes in ruminants. The authors discussed 2 situations known to affect gut function and integrity: the transition period from pregnancy to lactation and heat stress. They proposed a causality link between compromised gastrointestinal tract function and integrity to periparturient ketosis and heat stress damages. Their research suggests that transition period, exposure to heat stress, or restricted feed intake triggers intestinal permeability (i.e., leaky gut), leading to LPS uptake and, consequently, systematic inflammation, which in turn affects insulin signaling and glucose and fat metabolism. Following this argument, gut-targeting interventions could mitigate periparturient ketosis and susceptibility to heat stress in dairy cow.

The fourth speaker, Sordillo (2016), emphasized that understanding of the nutritional factors that influence immunity is critical to optimize nutrient conversion. In addition, dietary ingredients fed in deficiency or excess can lead to systemic inflammation, immune dysfunction, and, eventually, loss of productivity. Therefore, research on the gut constitutes an opportunity because, besides being a barrier and an endocrine organ, it is a primary lymphoid organ able to mount an immune response; likewise, its mucosa is an accessible target for dietary manipulations that may affect the gut per se as well as affect distal mucosal surfaces (Gill et al., 2010), contributing to amelioration of the cows gut health and overall health.

Dietary ingredients act as signals to multiple systems, and these signals affect dairy cow health, performance, and productivity. Building on this observation, the last speaker, Bradford et al. (2016), contrasted the classical task of nutritionists (i.e., meet nutritional requirements of the target animal) to their possible enhanced future role, which would consist of utilizing such dietary signals to influence metabolism, endocrinology, immunity, physiology, and, eventually, productivity.

In conclusion, progress in biological science has opened avenues for animal nutrition. Besides nutritive equilibrium, the diet controls immunity, gut microflora development, and neuroendocrine processes. This gut triad dramatically affects the productive outcome of a farm animal, which underlines the importance of shifting the paradigm in feed science. Of special interest is ruminant nutrition; "The rumen and beyond" symposium exposed scientists to various disciplines and highlighted the fact molecular and physiological development of the ruminant intestine and its function (e.g., sensory organ, immune surface) are under-researched areas that have potential impact on animal health and performance. This is all the more pertinent considering that economically important problems (e.g., heat stress response, ketosis) have now been linked to the gut, revealing an unsuspected yet critical role for this organ in dairy production. Efforts to understand the ways to manipulate the dairy cow gut response to various physiological, dietary, and environmental challenges will certainly uncover a new frontier for progress.

\section{REFERENCES}

Baumgard, L. H., S. K. Stoakes, M. Abuajamieh, and R. P. Rhoads. 2015. The contribution of the lower gut to altered nutrient partitioning during stress. J. Dairy Sci. 98(Suppl. 2):560-561. (Abstr.)

Bradford, B. J., K. Yuan, and C. Ylioja. 2016. Managing complexity: Dealing with systemic cross-talk in bovine physiology. J. Dairy Sci. 99:4983-4996.

Furness, J. B., L. R. Rivera, H. J. Cho, D. M. Bravo, and B. Callaghan. 2013. The gut as a sensory organ. Nat. Rev. Gastroenterol. Hepatol. 10:729-740.

Gill, N., M. Wlodarska, and B. B. Finlay. 2010. The future of mucosal immunology: Studying an integrated system-wide organ. Nat. Immunol. 11:558-560.

Sordillo, L. M. 2016. Nutritional strategies to optimize dairy cattle immunity. J. Dairy Sci. 99:4967-4982.

Steele, M. A., G. B. Penner, F. Chaucheyras-Durand, and L. Guan. 2016. Development and physiology of the rumen and the lower gut: Targets for improving production efficiency. J. Dairy Sci. 99:4955-4966.

VandeHaar, M. J., L. E. Armentano, K. Weigel, D. M. Spurlock, R. J. Tempelman, and R. Veerkamp. 2016. Harnessing the physiology of the modern dairy cow to continue improvements in feed efficiency. J. Dairy Sci. 99:4941-4954. 